\title{
Difference in Injury of the Corticospinal Tract and Spinothalamic Tract in Patients with Putaminal Hemorrhage
}

\author{
Sung Ho Jang', Jeong Pyo Seo ${ }^{2}$ \\ 'Department of Physical Medicine and Rehabilitation, College of Medicine, Yeungnam University, Daegu; ${ }^{2}$ Department of Physical Therapy, College \\ of Health Sciences, Dankook University, Cheonan, Korea
}

Purpose: We investigated the difference in injury of the corticospinal tract (CST) and the spinothalamic tract (STT) in patients with putaminal hemorrhage, using diffusion tensor tractography (DTT).

Methods: Thirty one consecutive patients with PH and 34 control subjects were recruited for this study. DTT scanning was performed at early stage of PH (7-63 days), and the CST and ST were reconstructed using the Functional Magnetic Resonance Imaging of Brain (FMRIB) Software Library program. Injury of the CST and ST was defined in terms of the configuration or abnormal DTT parameters was more than 2 standard deviations lower than that of normal control subjects.

Results: Among 31 patients, all 31 patients (100\%) had injury of the CTS, whereas 25 patients (80.6\%) had injury of the ST: the incidence of CST injury was significantly higher than that of ST $(p<0.05)$. In detail, $20(64.5 \%)$ of 31 patients showed a discontinuation of the CST in the affected hemisphere; in contrast, 14 patients (45.2\%) of 31 patients showed a discontinuation of the ST in the affected hemisphere. Regarding the FA value, 6 (19.4\%) of 31 patients and 2 (6.4\%) of 31 patients were found to have injury in the CST and ST, respectively. In terms of the fiber number, the same injury incidence was observed in 11 patients (35.5\%) in both the CST and ST.

Conclusion: The greater vulnerability of the CST appears to be ascribed to the anatomical characteristics; the CST is located anteriorly to the center of the putamen compared with the ST.

Keywords: Stroke, Putaminal hemorrhage, Corticospinal tract, Spinothalamic tract, Diffusion tensor tractography.

\section{INTRODUCTION}

The putamen is the most common area of spontaneous intracerebral hemorrhage (ICH), comprising $48-67 \%$ of all spontaneous ICH. ${ }^{1}$ Hemiplegia and hemisensory deficit, the main clinical features following putaminal hemorrhage $(\mathrm{PH})^{2}$, are related to the anatomical characteristics of the putamen, in which the neural tracts for sensori-motor function, such as the corticospinal tract (CST) and the spinothalamic tract (STT) are located near the putamen. ${ }^{3}$ In planning the scientific rehabilitation strategy for stroke patients, precise elucidation of the presence or severity of neural injury is mandatory because the physiatrist can assume the recovery mechanism, the recovery course and the final outcome based on the information on the neural injury. However, little is known about this topic in patients with $\mathrm{PH}$.

In the human brain, the CST is involved in control of movements of distal extremities, particularly fine-motor movements of the hand. ${ }^{4}$ The spinothalamic tract is responsible for pain and body temperature. ${ }^{5,6}$ In addition, the STT has been considered as the neural tract for the pathogenesis of central pain following brain injury. ${ }^{7}$ Considering the anatomy of the CST and STT around the putamen, because the CST is located closer to the center of the putamen than the STT, we hypothesized that the CST might be more vulnerable than the STT in patients with $\mathrm{PH}^{3}$

In this study, using diffusion tensor tractography (DTT), we attempted to investigate the difference in injury of the CST and STT in patients with $\mathrm{PH}$.
Received Nov 4, 2019 Revised Dec 4,2019

Accepted Dec 27, 2019

Corresponding author Jeong Pyo Seo

E-mail raphael0905@hanmail.net
Copylight (C2019 The Korean Society of Physical Therapy

This is an Open Access article distribute under the terms of the Creative Commons Attribution Non-commercial License (Http:// creativecommons.org/license/by-nc/4.o.) which permits unrestricted non-commercial use, distribution, and reproduction in any medium, provided the original work is properly cited. 


\section{METHODS}

\section{Subjects}

Thirty one patients with ICH (males: 22, females: 9, mean age: 48.8 years, range: 27 to 64 years) and 34 age-sex matched normal healthy control subjects (21 males, 13 females; mean age, 42.8 years, range: 27 to 64 years) with no history of neurological or psychiatric disorder were recruited for this study. Thirty one consecutive patients with ICH were recruited according to the following criteria: (1) firstever stroke, (2) age: 20-65 years, (3) DTI scanning was performed at early stage of ICH: 1-10 weeks after onset, and (4) hemorrhage confined at the level of the basal ganglia, and confirmed by a neuroradiologist. This study was conducted retrospectively and was approved by the institutional review board at our hospital.

\section{Diffusion tensor imaging}

DTI data were acquired at an average of 23 days after onset (7-63 days after onset) using a 6-channel head coil on a 1.5 T Philips Gyroscan Intera (Philips, Ltd, Best, The Netherlands) with single-shot echo-planar imaging (EPI). For each of the 32 non-collinear, diffusion-sensitizing gradients, 67 contiguous slices were acquired parallel to the anterior commissure-posterior commissure line. Imaging parameters were as follows: acquisition matrix $=96 \times 96$, reconstructed to matrix $=192 \times 192$, field of view $=240 \times 240 \mathrm{~mm}^{2}, \mathrm{TR}=$ $10.398 \mathrm{~ms}, \mathrm{TE}=72 \mathrm{~ms}$, parallel imaging reduction factor (SENSE factor $)=2$, EPI factor $=59, \mathrm{~b}=1,000 \mathrm{~s} / \mathrm{mm}^{2}, \mathrm{NEX}=1$, slice gap $=0$ $\mathrm{mm}$ and a slice thickness of $2.5 \mathrm{~mm}$.

Eddy current-induced image distortions were removed using affine multi-scale two-dimensional registration at the Oxford Centre for Functional Magnetic Resonance Imaging of Brain (FMRIB) Software Library (FSL: www.fmrib.ox.ac.uk/fsl). ${ }^{8}$ DTI-Studio software (CMRM, Johns Hopkins Medical Institute, Baltimore, MD, USA) was used for evaluation of the CST and STT. ${ }^{9}$ For reconstruction of the CST, the first ROI was placed on the anterior blue por-

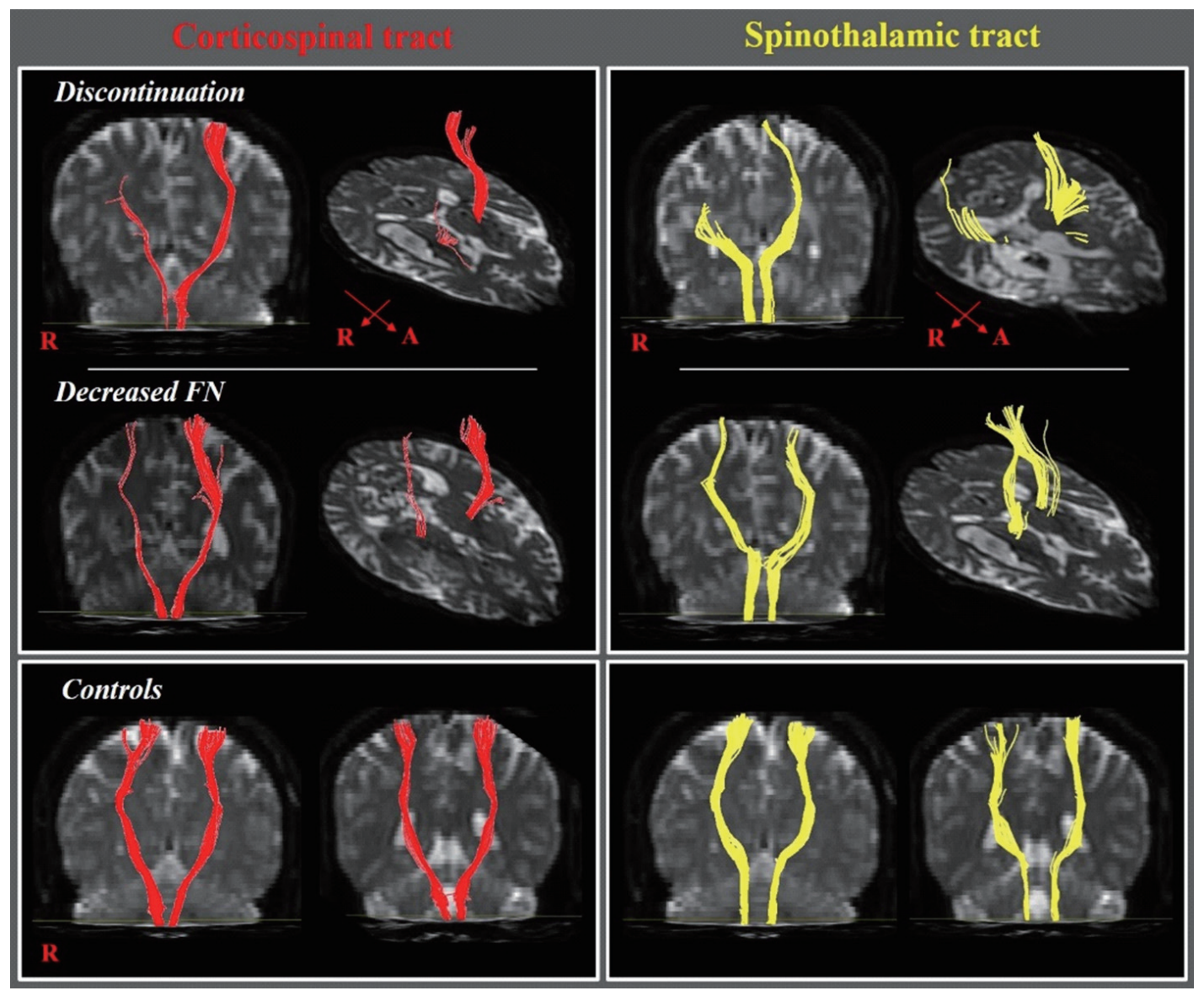

Figure 1. Results of diffusion tensor tractography (DTT) images of the corticospinal tract (CST) and the spinothalamic tract (STT). (A) Discontinuation of integrity of the CST and the STT. (B) Decreased fiber numbers of the CST and the STT. (C) DTT images of the CST and STT in control subjects. 
tion of the upper pons on the axial image of the color map, and the second ROI was placed on the anterior blue portion of the lower pons on the axial image of the color map. ${ }^{10}$ For analysis of the STT, the first ROI was placed on the postero-lateral medulla on an axial slice, and the second ROI was placed on the ventro-postero-lateral nucleus of the thalamus on the axial image of the color map. ${ }^{11}$ Fiber tracking was started at the seed voxels with a fractional anisotropy (FA) $>0.2$ and ended at the voxels with a fiber assignment of $>0.2$ and a tract turning-angle of $<60$ degrees. DTT parameters (fractional anisotropy [FA] and fiber number) of the CST and the STT were measured in both hemispheres. DTT parameters showing a deviation of more than two standard deviations of that of normal control values were defined as injury, and discontinuation of the CST and STT was defined as an injury.

The hematoma volume was measured on T2-weighted MRI images through a picture-archived communication system (PACS, Marotech, Korea) at the time of DTI scanning. Maximum width $(\mathrm{X})$, length $(\mathrm{Y})$, and height $(\mathrm{Z})$ of the lesion were measured at the level where hemorrhage of the putamen was clearly seen. ${ }^{12}$ ICH volume was calculated according to the formula (Figure 1):

$$
\text { Hematoma volume }(\mathrm{mV})=\frac{4}{3} \times \frac{1}{16} \times \Pi \times \mathrm{X}(\mathrm{cm}) \times \mathrm{Y}(\mathrm{cm}) \times \mathrm{Z}(\mathrm{cm}) .
$$

\section{Statistical analysis}

SPSS software (v.15.0, SPSS, Chicago, IL, USA) was used for data analysis. The chi-square test was used for determination of the differences in incidence of injury between the CST and STT. Correlations between the hematoma volume, and FA value and fiber number of the CST and the STT in the affected hemisphere were deter- mined using Pearson's correlation test. The significant level of the $\mathrm{p}$ value was set at 0.05 .

\section{RESULTS}

Data on demographic and DTT parameters of the patients and control subjects are summarized in Table 1 . Of 31 patients, all 31 patients (100\%) had injury of the CST, whereas 25 patients $(80.6 \%)$ had injury of the STT: the incidence of CST injury was significantly higher compared with that of the STT $(\mathrm{p}<0.05)$. In detail, $20(64.5 \%)$ of 31 patients showed a discontinuation of the CST in the affected hemisphere; in contrast, 14 patients (45.2\%) of 31 patients showed a discontinuation of the STT in the affected hemisphere. Regarding the FA value, 6 (19.4\%) of 31 patients and $2(6.4 \%)$ of 31 patients were found to have injury in the CST and STT, respectively. In terms of the fiber number, the same injury incidence was observed in 11 patients (35.5\%) in both the CST and STT (Table 2). On the other hand, no correlation was found between the hematoma volume, and FA value or the fiber number $(\mathrm{p}>0.05)$.

Table 2. Distribution of patients according to injury types between the corticospinal tract and spinothalamic tract

$(n=31)$

\begin{tabular}{lcc}
\hline & CST (\%) & ST (\%) \\
\hline Total injury incidence & $31(100)$ & $25(80.6)$ \\
Discontinuation & $20(64.5)$ & $14(45.2)$ \\
Injury of FA & $6(19.4)$ & $2(6.5)$ \\
Injury of FN & $11(35.5)$ & $11(35.5)$ \\
Normal & $0(0)$ & $6(19.4)$ \\
\hline
\end{tabular}

CST: corticospinal tract, STT: spinothalamic tract, FA: fractional anisotropy, FN: fiber number.

Table 1. Demographic and diffusion tensor tractography data of the patients and control subjects

\begin{tabular}{|c|c|c|c|}
\hline & \multicolumn{2}{|c|}{ Patient group $(n=31)$} & Control group $(n=34)$ \\
\hline Age (yr) & \multicolumn{2}{|c|}{$48.8( \pm 9.9)$} & $42.8( \pm 10.5)$ \\
\hline Sex (male/female) & \multicolumn{2}{|c|}{$22 / 9$} & $21 / 13$ \\
\hline Duration after onset (day) & \multicolumn{2}{|c|}{$22.7( \pm 15.8)$} & \\
\hline Hematoma volume (mV) & \multicolumn{2}{|c|}{$4.12( \pm 2.90)$} & \\
\hline Fractional anisotropy & Affected & Unaffected & \\
\hline Corticospinal tract & $0.46( \pm 0.07)$ & $0.55( \pm 0.03)$ & $0.57( \pm 0.02)$ \\
\hline Spinothalamic tract & $0.47( \pm 0.04)$ & $0.51( \pm 0.03)$ & $0.51( \pm 0.03)$ \\
\hline Fiber number & Affected & Unaffected & \\
\hline Corticospinal tract & $642.8( \pm 497.6)$ & $1,710.8( \pm 344.6)$ & $1,873.0( \pm 206.6)$ \\
\hline Spinothalamic tract & $672.3( \pm 395.7)$ & $1,443.1( \pm 368.8)$ & $1,378.3( \pm 197.8)$ \\
\hline
\end{tabular}

Values indicate mean ( \pm standard deviation). 


\section{DISCUSSION}

In the current study, using DTT, we investigated the characteristics of injury of the CST and STT in 31 consecutive patients with PH in terms of presence of discontinuation on DTT and DTT parameters (the FA and fiber number). We found that the total incidence of injury was higher in the CST (100\%) than the STT (80.6\%): in detail, discontinuation on DTT: the CST (64.5\%) and the STT (45.2\%), presence of injury in the FA value: the CST (19.4\%) and the STT (6.5\%), and presence of injury in the fiber number: the CST (35.5\%) and the STT (35.5\%).

The FA value is the most commonly used DTT parameter indicating the degree of directionality of microstructures, such as axons, myelin, and microtubules. ${ }^{13,14}$ The fiber number indicates the total number of neural fibers in a neural tract. ${ }^{15}$ Therefore, the decrement of the FA value or the fiber number of the CST or STT indicates an injury of the neural tract which appears to indicate mild or partial injury because this type means the decrement of FA value or fiber number with preservation of the integrity of the CST or STT. By contrast, discontinuation of the CST or STT tract appears to suggest more severe or complete injury of each neural tract because this type indicates discontinuation of a neural tract. Therefore, based on our results, it appears that the CST is more vulnerable than the STT in terms of the incidence and severity of injury following $\mathrm{PH}$. The greater vulnerability of the CST appears to be ascribed to the anatomical characteristics of the CST and STT, the CST is located anteriorly to the center of the putamen compared with the STT. ${ }^{3}$

In conclusion, we investigated the characteristics of injury of the CST and STT in 31 consecutive patients with PH and found that the CST was more vulnerable than the STT in terms of incidence and severity of injury. To the best of our knowledge, this is the first study to investigate the characteristics of injury of the CST and STT in patients with $\mathrm{PH}$. We believe that these results will be helpful to rehabilitation clinicians in clinical practice such as set a strategy of treatment for patients with PH. However, some limitations should be considered in interpretation of this study. First, the small number of subjects; second, the patients were recruited among those with $\mathrm{PH}$ who had been admitted for rehabilitation. Therefore, it is possible that among all patients with $\mathrm{PH}$, we recruited patients with severe clinical features. Third, due to hematoma or peri-hematomal edema, the neural tracts can be underestimated on DTT ${ }^{16,17}$ Last, because this study was conducted retrospectively, we could not confirm the final outcome of these neural tracts at chronic stage. Therefore, further studies recruiting larger numbers of patients and follow up DTT at chronic stage should be encouraged.

\section{ACKNOWLEDGEMENTS}

This work was supported by the National Research Foundation of Korea (NRF) grant funded by the Korean Government(MSIP) (No. 2018R1A2B6000996).

\section{REFERENCES}

1. Inagawa T, Ohbayashi N, Takechi A et al. Primary intracerebral hemorrhage in izumo city, japan: Incidence rates and outcome in relation to the site of hemorrhage. Neurosurgery. 2003;53(6):1283-97.

2. Bogousslavsky J, Caplan LR. Stroke syndromes. 2nd. Cambridge, New York, Cambridge University Press, 2001.

3. Jang SH. A review of corticospinal tract location at corona radiata and posterior limb of the internal capsule in human brain. NeuroRehabilitation. 2009;24(3):279-83.

4. Jang SH. The corticospinal tract from the viewpoint of brain rehabilitation. J Rehabil Med. 2014;46(3):193-9.

5. Afifi AK, Bergman RA. Functional neuroanatomy: Text and atlas. 2nd. New York, Lange Medical Books/McGraw-Hill, 2005.

6. Mendoza JE, Foundas AL. Clinical neuroanatomy: A neurobehavioral approach. New York, Springer, 2007.

7. Kim JH, Ahn SH, Cho YW et al. The relation between injury of the spinothalamocortical tract and central pain in chronic patients with mild traumatic brain injury. J Head Trauma Rehabil. 2015;31(6):E40-6.

8. Smith SM, Jenkinson M, Woolrich MW et al. Advances in functional and structural MR image analysis and implementation as FSL. Neuroimage. 2004;23 Suppl 1:S208-19.

9. Jiang H, van Zijl PC, Kim J et al. Dtistudio: Resource program for diffusion tensor computation and fiber bundle tracking. Comput Methods Programs Biomed. 2006;81(2):106-16.

10. Jang SH. Somatotopic arrangement and location of the corticospinal tract in the brainstem of the human brain. Yonsei Med J. 2011;52(4):5537.

11. Hong JH, Son SM, Jang SH. Identification of spinothalamic tract and its related thalamocortical fibers in human brain. Neurosci Lett. 2010; 468(2):102-5.

12. Kwak R, Kadoya S, Suzuki T. Factors affecting the prognosis in thalamic hemorrhage. Stroke. 1983;14(4):493-500.

13. Mori S, Crain BJ, Chacko VP et al. Three-dimensional tracking of axonal projections in the brain by magnetic resonance imaging. Ann Neurol. 1999;45(2):265-9.

14. Assaf Y, Pasternak O. Diffusion tensor imaging (DTI)-based white matter mapping in brain research: A review. J Mol Neurosci. 2008;34(1):5161. 
15. Jang SH, Jang WH. Change of the corticospinal tract in the unaffected hemisphere by change of the dominant hand following stroke: A cohort study. Medicine (Baltimore). 2016;95(6):e2620.

16. Parker GJ, Alexander DC. Probabilistic anatomical connectivity derived from the microscopic persistent angular structure of cerebral tissue. Philos Trans R Soc Lond B Biol Sci. 2005;360(1457):893-902.

17. Yamada K, Sakai K, Akazawa K et al. Mr tractography: A review of its clinical applications. Magn Reson Med Sci. 2009;8(4):165-74. 